\title{
Clinical Significance of Tryptophan Metabolism in the Nontumoral Hemisphere in Patients with Malignant Glioma
}

\author{
David O. Kamson ${ }^{1,2}$, Tiffany J. Lee ${ }^{1}$, Kaushik Varadarajan ${ }^{1}$, Natasha L. Robinette ${ }^{3,4}$, Otto Muzik ${ }^{1-3,5}$, \\ Pulak K. Chakraborty ${ }^{1,3}$, Michael Snyder ${ }^{4}$, Geoffrey R. Barger ${ }^{4,5}$, Sandeep Mittal ${ }^{4,6,7}$, and Csaba Juhász ${ }^{1,2,4,5}$ \\ ${ }^{I}$ PET Center and Translational Imaging Laboratory, Children's Hospital of Michigan, Detroit, Michigan; ${ }^{2}$ Department of Pediatrics, \\ Wayne State University, Detroit, Michigan; ${ }^{3}$ Department of Radiology, Wayne State University, Detroit, Michigan; ${ }^{4}$ Karmanos Cancer \\ Institute, Detroit, Michigan; ${ }^{5}$ Department of Neurology, Wayne State University, Detroit, Michigan; ${ }^{6}$ Department of Neurosurgery, \\ Wayne State University, Detroit, Michigan; and ${ }^{7}$ Department of Oncology, Wayne State University, Detroit, Michigan
}

$\mathrm{a}^{-11} \mathrm{C}$-methyl-L-tryptophan (AMT) PET allows evaluation of brain serotonin synthesis and can also track upregulation of the immunosuppressive kynurenine pathway in tumor tissue. Increased AMT uptake is a hallmark of World Health Organization grade III-IV gliomas. Our recent study also suggested decreased frontal cortical AMT uptake in glioma patients contralateral to the tumor. The clinical significance of extratumoral tryptophan metabolism has not been established. In the present study, we investigated clinical correlates of tryptophan metabolic abnormalities in the nontumoral hemisphere of glioma patients. Methods: Standardized AMT uptake values (SUVs) and the uptake rate constant of AMT $(\mathrm{K}[\mathrm{mL} / \mathrm{g} / \mathrm{min}]$, a measure proportional to serotonin synthesis in nontumoral gray matter) were quantified in the frontal and temporal cortex and thalamus in the nontumoral hemisphere in 77 AMT PET scans of 66 patients (41 men, 25 women; mean age \pm SD, $55 \pm 15$ y) with grade III-IV gliomas. These AMT values were determined before treatment in 35 and after treatment in 42 patients and were correlated with clinical variables and survival. Results: AMT uptake in the thalamus showed a moderate age-related increase before treatment (SUV, $r=0.39, P=0.02)$ but decrease after treatment $(\mathrm{K}, r=-0.33$, $P=0.057)$. Women had higher thalamic SUVs before treatment $(P=0.037)$ and higher thalamic $(P=0.013)$ and frontal cortical $\mathrm{K}$ values $(P=0.023)$ after treatment. In the posttreatment glioma group, high thalamic SUVs and high thalamocortical SUV ratios were associated with short survival in Cox regression analysis. The thalamocortical ratio remained strongly prognostic $(P<0.01)$ when clinical predictors, including age, glioma grade, and time since radiotherapy, were entered in the regression model. Long interval between radiotherapy and posttreatment $A M T$ PET as well as high radiation dose affecting the thalamus were associated with lower contralateral thalamic or cortical AMT uptake values. Conclusion: These observations provide evidence for altered tryptophan uptake in contralateral cortical and thalamic brain regions in glioma patients after initial therapy, suggesting treatment effects on the serotonergic system. Low thalamic tryptophan uptake appears to be a strong, independent predictor of long survival in patients with previous glioma treatment.

Received Apr. 3, 2014; revision accepted Jul. 29, 2014

For correspondence or reprints contact: Csaba Juhász, PET Center and Translational Imaging Laboratory, Children's Hospital of Michigan, 3901 Beaubien St., Detroit, MI 48201.

E-mail: juhasz@pet.wayne.edu

Published online Sep. 4, 2014.

COPYRIGHT (C 2014 by the Society of Nuclear Medicine and Molecular Imaging, Inc.
Key Words: glioma; brain; tryptophan metabolism; PET; survival

J Nucl Med 2014; 55:1605-1610

DOI: 10.2967/jnumed.114.141002

A MT ( $\alpha-{ }^{11} \mathrm{C}-$ methyl-L-tryptophan) is a radiotracer originally developed to assess brain serotonin synthesis using PET (1-4). Moreover, under pathologic conditions, AMT PET can also track the upregulation of the immunosuppressive kynurenine pathway (leading to enhanced conversion of tryptophan to kynurenine metabolites) in tumor tissue and epileptic foci (4-8). Increased tumoral AMT uptake is a hallmark of grade III-IV gliomas $(9,10)$ and is also a strong imaging marker of poor survival when detected after initial glioma treatment (surgery and chemoradiation) (11).

Although several amino acid PET tracers have been used to characterize brain tumors (9-13), only AMT PET has the additional ability to study extratumoral cerebral tryptophan metabolism and serotonergic abnormalities. In healthy adults, AMT PET studies of brain serotonin synthesis revealed some sex differences without a clear age effect (14-16). AMT PET has also been used to study focal and global serotonergic brain abnormalities in autism, migraine, and various psychiatric conditions (3,17-19). Until recently, however, no studies addressed potential abnormalities and clinical correlates of brain tryptophan metabolism in neurooncologic patients. Recently, our group reported decreased frontal cortical AMT uptake in treatment-naïve patients with brain tumors, as compared with healthy control subjects (20), but the potential clinical significance of extratumoral abnormalities of brain tryptophan uptake remained to be clarified.

In the present study, we assessed brain regional changes of AMT uptake in a large cohort of patients evaluated with gliomas. We hypothesized that cortical or thalamic AMT uptake contralateral to the glioma will be related to clinical variables, such as sex, age, glioma histologic grade, treatment status (pre- vs. posttreatment), or radiation effects. We also explored a potential association between AMT uptake in nontumoral contralateral brain regions and survival.

\section{MATERIALS AND METHODS}

\section{Subjects}

Sixty-six adult patients (41 men, 25 women; mean age \pm SD, $55 \pm$ $15 \mathrm{y})$, diagnosed with unilateral World Health Organization grade III-IV 
glioma (21 grade III, 45 grade IV), underwent AMT PET imaging. Thirty-five patients were studied before treatment, within $3 \mathrm{wk}$ before initial tumor resection. Forty-two patients underwent PET after initial treatment (i.e., surgery, followed by chemoradiation) because of suspicion of glioma recurrence based on serial MR images or clinical progression. Patients with poor performance status (such as a Karnofsky performance score of 50 or below) were not included. Most pretreatment patients were on prophylactic antiepileptic medication (levetiracetam in most cases), and 12 patients had at least 1 documented clinical seizure and epileptiform activity on electroencephalography before initial treatment. All these patients underwent surgical tumor resection, followed by chemoradiation. Posttreatment patients were taking temozolomide after the completion of radiotherapy until radiographic tumor progression. The time between completion of initial radiotherapy and the PET scan was below $5 \mathrm{y}$ in all but 2 patients $(2 \mathrm{mo}-4.5 \mathrm{y}$; median, $1.2 \mathrm{y}$ ); 2 patients (with grade III glioma) underwent their initial radiotherapy 6.7 and 11.6 y before the AMT PET. Second-line therapy after the AMT PET scan included bevacizumab with or without irinotecan $(n=16)$ or second surgery $(n=14)$. Eleven patients underwent both pre- and posttreatment AMT PET scans; thus, 77 AMT PET scans were analyzed. Of the patients with grade III glioma, 6 had $1 \mathrm{p} / 19 \mathrm{q}$ deletion; isocitrate dehydrogenase 1 mutation was not available in all patients, particularly those studied several years ago. The study has been approved by the Institutional Review Board of Wayne State University, and all subjects signed a written informed consent form.

\section{AMT PET Scanning Protocol}

AMT PET images were acquired using an EXACT/HR whole-body positron emission tomograph (Siemens Medical Systems). AMT was synthesized using a high-yield procedure (21). The procedure for AMT PET scanning has been described in detail previously $(9,22,23)$. Briefly, after $6 \mathrm{~h}$ of fasting $3.7 \mathrm{MBq}$ of AMT per kilogram were injected. Coinciding with tracer injection, a dynamic PET scan of the heart was acquired to obtain the blood input function from the left cardiac ventricle. The blood input function was continued using venous blood samples $(0.5 \mathrm{~mL} / \mathrm{sample}$, collected at 20,30, 40,50, and $60 \mathrm{~min}$ after AMT injection) (9). At 25 min after tracer injection, a dynamic emission scan of the brain $(7 \times 5 \mathrm{~min})$ was obtained. Measured attenuation correction, scatter, and decay correction were applied. All images were reconstructed with filtered backprojection using a Hanning filter, yielding images with an in-plane resolution of $7.5 \pm 0.4 \mathrm{~mm}$ in full width at half maximum and $7.0 \pm 0.5 \mathrm{~mm}$ in full width at half maximum in the axial direction. Primary image analysis was performed using AMT standardized uptake value (SUV) images, because venous blood samples for blood input function were not available in 9 patients. The SUV was calculated by dividing the average tracer concentration in tissue at 30-55 min by the ratio of injected activity and patient weight. AMT uptake was also quantified by Patlak graphical analysis (24) using the blood input function and tissue time-activity curves, yielding the uptake rate constant $\mathrm{K}$ for AMT $(\mathrm{mL} / \mathrm{g} / \mathrm{min})(9,22$, $23)$ in 68 PET scans. The AMT K value reflects the unidirectional uptake of tracer into tissue, which is proportional to the metabolism of tryptophan via serotonin synthesis in nonlesional brain (4).

\section{MR Imaging Protocol}

T1-weighted postcontrast images were used as anatomic guides for the segmentation of the regions of interest (ROIs). MR imaging was performed on a MAGNETOM Trio TIM 3.0-T scanner (Siemens Medical Solutions), a Signa HDxt 3.0-T scanner (GE Healthcare), or an Achieva TX 3.0-T scanner (Philips), using similar parameters on all scanners.

\section{Analysis of Regional AMT Uptake}

The 3-dimensional Slicer 3.6.3 software suite (Brigham and Women's Hospital, Inc.) was used for image analysis $(23,25)$. The MR (T1-weighted before and after contrast, fluid-attenuated inversion recovery) and PET images were coregistered using the Fast Rigid Registration module (26). Thalamic and cortical ROIs contralateral to the tumor were segmented manually using the axial view of the PET/MR imaging fusion images (Fig. 1). The ROIs included gray matter voxels with AMT uptake: a single plane for the thalamus with the largest axial thalamic diameter and multiple planes (1-2 planes apart) for the cortical ROIs including the frontal ( 3 planes) and temporal lobes ( 2 planes). None of the thalamic or cortical ROIs showed abnormal contrast-enhancement or fluid-attenuated inversion recovery signal on coregistered MR images. The parietal cortex, with uptake similar to the frontal cortex, was not separately evaluated. Likewise, the occipital cortex, which often shows high physiologic uptake, was also not included in the analyses. AMT SUVs for each ROI were quantified using an approach previously described (23). Mean SUVs were obtained for all defined regions. Also, thalamocortical (i.e., thalamus-to-cortex) ratios were calculated using the average values of all cortical ROIs. Mean tumor SUV, a parameter with strong prognostic value for survival in our recent study (11), was also measured, as described previously $(10,11,23)$. These measurements were also applied for the AMT K values; however, most clinical correlates were less or not significant. For simplicity, with the exception of sex and age differences, only results for AMT SUV are reported.

\section{Statistical Analysis}

First, age-related differences in AMT SUVs and $\mathrm{K}$ values were assessed in the pre- and posttreatment subgroups using Pearson correlations. Differences in cortical and thalamic AMT uptake, related to sex and glioma histologic grade (the latter obtained from the initial surgery), were assessed using unpaired $t$ tests.

Survival analyses were performed for the pretreatment and posttreatment groups separately. Survival was calculated from the time of the AMT PET scan. First, we used univariate Cox regression analyses with potential prognostic variables_-including clinical variables such as age, glioma histologic grade, time between initial therapy and AMT PET (in the posttreatment group), second-line chemotherapy, or repeated surgery after PET - and with PET variables (thalamic and cortical values as well as thalamocortical ratios). PET variables found to be significant predictors in the univariate analyses were then entered in multivariate Cox regression analyses along with clinical predictors that showed significance or a trend $(P<0.1)$ in the univariate analysis. A similar analysis was also performed to determine the prognostic value of AMT uptake when the mean tumor SUV (a previously reported predictor of posttreatment survival (11)) was also included as a predictor.

To explore whether radiation could have affected nontumoral AMT uptake, we correlated AMT SUVs in the nontumoral hemisphere and the time elapsed between completion of radiation and the posttreatment PET scan using Pearson correlation; we have also examined the scatterplots for nonlinearity. Because the thalamus was often located

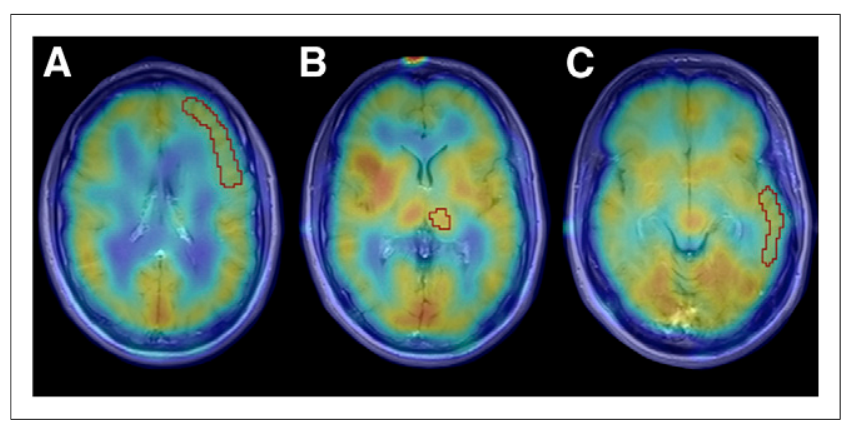

FIGURE 1. AMT PET/MR fusion images of patient with right insular glioma. Contralateral frontal (A), thalamic (B), and temporal (C) ROls used for analyses are outlined in red. 
within the field of radiation, we also measured the radiation dose absorbed by the thalamus of the nontumoral hemisphere of high-grade glioma patients who received radiation therapy in our institution before the AMT PET $(n=23)$. We used the Eclipse Treatment Planning Workstation 8.9 (Varian Medical Systems) to load the original radiation plans and segment the thalami, and then we quantified the maximum radiation dose absorbed in this region. For the patients whose thalamus received at least $20 \%$ of the total tumoral radiation (i.e., $>10-12$ Gy; $n=20$ ), we correlated the thalamic dose with the thalamic and cortical AMT parameters using Pearson correlation. We also evaluated whether the radiation dose to the thalamus had an effect on survival using a Cox regression analysis in this subgroup.

Finally, we compared pre- and posttreatment cortical and thalamic AMT SUV in the 11 patients who were scanned both before and after glioma treatment, using the Wilcoxon test; because these comparisons showed no significant results (or even trends), these data are not included in the "Results" section. All statistical analyses were performed using SPSS Statistics 20.0 (SPSS). A $P$ value of less than 0.05 was considered statistically significant.

\section{RESULTS}

\section{Effects of Age, Sex, and Glioma Histologic Grade}

Pretreatment thalamic SUVs showed a moderate increase with age $(r=0.39, P=0.02)$, whereas the posttreatment group showed a trend for age-related decline in the thalamic $\mathrm{K}$ values $(r=-0.33$, $P=0.057$ ). In sex comparisons, AMT SUVs and $\mathrm{K}$ values were generally higher in women in both the cortex and the thalamus, with the following differences reaching statistical significance: thalamic SUV in the pretreatment group $(3.0 \pm 0.8$ vs. $2.5 \pm 0.5, P=0.037)$ as well as AMT K values in the thalamus $(P=0.013)$ and in the frontal cortex $(P=0.023)$ in the posttreatment group. Grade III versus IV glioma patients showed no thalamic or cortical AMT uptake differences in pretreatment patients. In the posttreatment group, thalamic AMT SUV was slightly higher in patients with grade IV than in those with grade III glioma $(3.0 \pm 0.7$ vs. $2.6 \pm 0.6, P=0.045)$.

\section{Thalamic and Cortical AMT Uptake and Survival in Patients with High-Grade Glioma}

In the pretreatment group of patients, none of the AMT PET parameters were prognostic for survival in Cox regression analyses. In the posttreatment group, univariate Cox regression analyses (Table 1) showed that high thalamocortical ratios (particularly values above 1.19; Fig. 2) and, to a lesser degree, high thalamic and frontal SUV were all prognostic for poor survival. Among the clinical predictors, higher glioma grade and short time between radiotherapy and AMT PET were prognostic for poor survival. Higher age was only marginally prognostic for poor survival. In multivariate Cox regression (Table 2), the thalamocortical SUV ratios remained strongly prognostic when age, glioma histologic grade, time between radiotherapy and PET, or mean tumoral SUV were added as a second (clinical) prognostic variable to the model. The thalamic SUVs remained significant when age, interval between radiotherapy, and PET but not when glioma grade or tumoral SUV were added to the model. The frontal SUVs were prognostic only when age was added to the model (Table 2).

\section{Relationship Between Radiation Exposure and Contralateral AMT Uptake}

In the whole group, both thalamic SUVs and thalamocortical SUV ratios showed a negative correlation with the time between radiotherapy, but this correlation was nonsignificant when linear (Pearson) correlation was applied; however, the scatterplots suggested a logarithmic relationship, which was significant for both thalamic SUVs $(r=0.33, P=0.035)$ and the thalamocortical ratios $(r=-0.44, P=0.004)$. This negative relation was also observed in the glioblastoma subgroup (thalamocortical: $r=0.46$, $P=0.011$; thalamus SUV: $r=-0.4, P=0.054$ ).

The maximum thalamic radiation doses (range, 1.3-60.9 Gy; mean, $44.0 \mathrm{~Gy}$ ) showed an inverse correlation with cortical and thalamic SUVs, which was significant only for the cortex (frontal: $r=-0.49$, $P=0.03$; temporal: $r=-0.45, P=0.047$; thalamus: $r=-0.29$;

TABLE 1

Results of Univariate Cox Regression Analyses for Potential Predictors of Survival in Posttreatment Group $(n=42)$

\begin{tabular}{|c|c|c|}
\hline Potential prognostic variables & $\mathrm{HR}(95 \% \mathrm{Cl})$ & $P$ \\
\hline \multicolumn{3}{|l|}{ AMT PET variables } \\
\hline Thalamic SUV & $2.23(1.3-3.8)$ & $0.004^{*}$ \\
\hline Frontal cortical SUV & $2.01(1.01-4.4)$ & $0.047^{\star}$ \\
\hline Temporal cortical SUV & $1.90(0.99-3.6)$ & 0.052 \\
\hline Thalamocortical SUV ratio & $2,544(43-149,205)$ & $<0.001^{*}$ \\
\hline Tumoral SUV & $1.57(1.18-2.10)$ & $0.002^{*}$ \\
\hline \multicolumn{3}{|l|}{ Clinical variables } \\
\hline Age (y) & $1.03(0.99-1.05)$ & 0.089 \\
\hline Glioma histologic grade (IV vs. III) & $31(4.0-243)$ & $0.001^{*}$ \\
\hline Time since radiotherapy $(\mathrm{y})$ & $0.50(0.30-0.83)$ & $0.008^{\star}$ \\
\hline Repeated surgery (yes vs. no) & $1.25(0.55-2.85)$ & 0.58 \\
\hline Second-line chemotherapy (yes vs. no) & $1.54(0.70-3.40)$ & 0.28 \\
\hline \multicolumn{3}{|c|}{$\begin{array}{l}{ }^{\star} P \text { value is significant. } \\
\mathrm{HR}=\text { hazard ratio; } \mathrm{Cl}=\text { confidence interval. } \\
\text { Both AMT PET variables and clinical predictors were tested for prediction of survival after PET scan. Note high HR and low } P \text { values for } \\
\text { lalamocortical ratios; extremely high } \mathrm{HR} \text { values partly reflect limited range of this variable (1.09-1.55 for this subgroup), thus, full unit } \\
\text { ffference (e.g., from } 1 \text { to } 2 \text { ) between } 2 \text { individuals would be associated with extremely different risk for death. }\end{array}$} \\
\hline
\end{tabular}




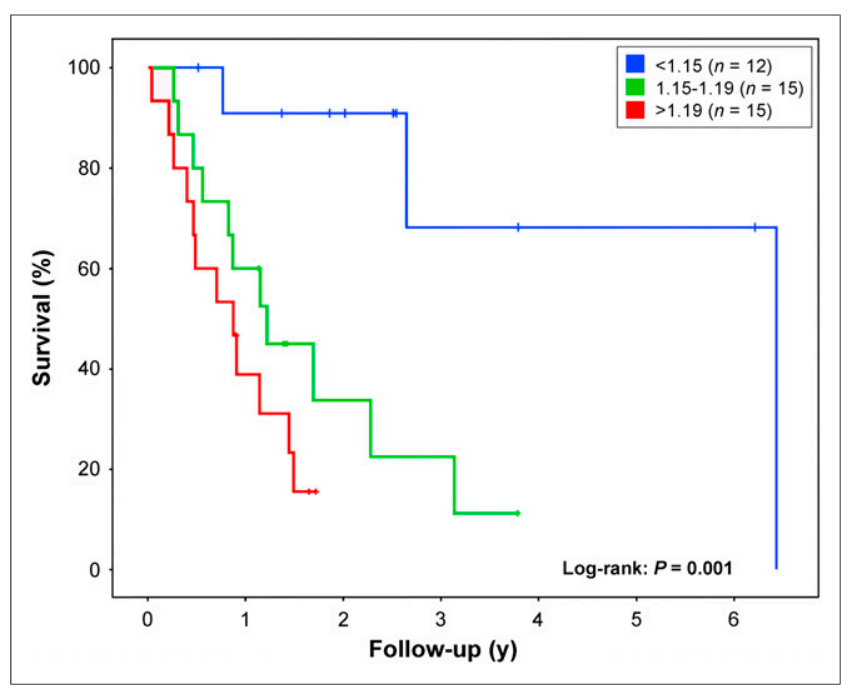

FIGURE 2. Prognostic value of contralateral thalamocortical AMT SUV ratios for survival in posttreatment group. Kaplan-Meier survival curves are shown for patients whose ratios were in bottom, middle, and upper tertile of group (total of 42 patients; subgroups have uneven numbers due to ties in values around tertile cutoff). Survival curves demonstrate that thalamocortical ratios below 1.15 were strongly prognostic for long survival, whereas middle values (1.15-1.19) provided moderate survival advantage over highest values ( 1.20 or above).

$P=0.2)$. Finally, thalamic radiation dose did not predict survival $(P=0.57)$.

\section{DISCUSSION}

This is the first study, to our knowledge, to explore clinical correlates of tryptophan uptake in extratumoral contralateral brain regions in patients with brain tumors. We found that most of the clinical correlates occurred in the posttreatment subgroup, suggesting that the findings may have been related to treatment effects. We also found evidence for potential radiation effects on the contralateral brain. Perhaps the most intriguing result is the prognostic value of thalamic and cortical SUVs and SUV ratios in posttreatment glioma patients.
The reason for the prognostic value of the thalamic and cortical SUV (and also SUV ratios) in the posttreatment group remains to be determined. Possible explanations include the presence of infiltrative glioma cells in distant brain regions (27) or upregulation of the inflammatory kynurenine pathway in these regions, perhaps induced by glioma-associated cytokines (28). In the ipsilateral hemisphere, we have recently shown that glioma-infiltrated brain can indeed show increased AMT uptake even if conventional MR imaging shows no abnormalities (10). Regardless of the mechanisms, the strong prognostic value of thalamic AMT SUV in the posttreatment group may indicate a link between extratumoral amino acid metabolism and survival. The association of high thalamocortical SUV ratios and short survival remained significant even when several clinical predictors or tumoral SUVs were entered in multivariate regressions. Thus, thalamic SUV and thalamocortical SUV ratio may complement other prognostic variables for predicting survival after initial treatment. The practical advantage of measuring extratumoral tracer uptake is that measurements in the contralateral hemisphere can be obtained in a more standardized way than measurements from heterogeneous posttreatment changes suspicious for tumor recurrence. SUV is a simple, practical uptake measure, which has been shown to correlate with AMT K values, although the latter parameter is more intimately related to serotonin synthesis. However, measurement of AMT K is not practical in the clinical setting because it requires invasive blood sampling.

In addition to the prognostic value discussed above, some interesting sex differences as well as effects of radiation on contralateral tryptophan uptake deserve discussion. The slightly higher values in women might be related to physiologic sex differences in brain serotonin synthesis. Indeed, a previous AMT PET study from our group reported $10 \%-20 \%$ higher serotonin synthesis rates in healthy women than men (2). A subsequent smaller study from the Montreal group, applying an objective, voxel-by-voxel comparison, found a more complex pattern, with region-specific sex differences (16). However, the same group published data from a larger healthy cohort $(n=59)$, in which men showed higher AMT trapping in multiple cortical regions with no subcortical differences (29). In the present study, the sex differences in AMT K values were more pronounced and widespread (present both in the thalamus and in the cortex) in the posttreatment group, possibly indicating serotonergic

TABLE 2

Results of Multivariate Cox Regression Analyses

\begin{tabular}{|c|c|c|c|c|c|c|c|c|}
\hline \multirow[b]{3}{*}{ AMT PET predictors } & \multicolumn{8}{|c|}{ Covariate added to Cox regression } \\
\hline & \multicolumn{2}{|l|}{ Age } & \multicolumn{2}{|c|}{ Glioma grade } & \multicolumn{2}{|c|}{$\begin{array}{l}\text { Time since } \\
\text { radiotherapy }\end{array}$} & \multicolumn{2}{|l|}{ Tumor SUV } \\
\hline & $\mathrm{HR}(95 \% \mathrm{Cl})$ & $P$ & $\mathrm{HR}(95 \% \mathrm{Cl})$ & $P$ & $\mathrm{HR}(95 \% \mathrm{Cl})$ & $P$ & $\mathrm{HR}(95 \% \mathrm{Cl})$ & $P$ \\
\hline Thalamic SUV & $2.2(1.3-3.8)$ & $0.005^{\star}$ & $1.6(0.9-2.9)$ & 0.1 & $1.9(1.1-3.3)$ & $0.015^{\star}$ & $1.7(0.9-3.2)$ & 0.1 \\
\hline Frontal SUV & $2.2(1.04-4.6)$ & $0.04^{*}$ & $1.5(0.7-3.3)$ & 0.29 & $1.9(0.95-3.9)$ & 0.07 & $1.4(0.6-3.4)$ & 0.37 \\
\hline $\begin{array}{l}\text { Thalamocortical } \\
\text { SUV ratio }\end{array}$ & $1776(25-122,730)$ & $0.001^{*}$ & $597(6.3-56,621)$ & $0.005^{\star}$ & $329(4-25,649)$ & $0.009^{*}$ & $506(4.9-51,784)$ & $0.008^{*}$ \\
\hline
\end{tabular}

${ }^{*} P$ value is significant.

$\mathrm{HR}=$ hazard ratio; $\mathrm{Cl}=$ confidence interval.

Clinical variables and mean tumoral SUV were entered as covariates for each of the contralateral AMT predictors found significant in univariate analysis. Hazard ratios are given for AMT PET predictors after addition of each covariate to model. Thalamocortical AMT SUV ratio remained highly prognostic for survival with each of the covariates. 
changes, especially in women, after glioma therapy. The clinical significance of these remains to be clarified. One plausible clinical implication of brain serotonergic changes involves anxiety and depression, common comorbidities in glioma patients $(30,31)$, with a potentially higher incidence in women (32). Thus, AMT PET could be a useful imaging modality to clarify the role of abnormal brain serotonin synthesis, and their sex differences, in glioma-associated depression.

The age-related results are partly consistent with a previous AMT PET study of healthy subjects showing no age-related changes in serotonin synthesis rates in the cortex or in the subcortical gray matter (14). In our present study, we also did not detect robust agerelated variations, except a mild thalamic increase in the pretreatment and decline in the posttreatment subgroup. One possible explanation is that treatment, which typically included both local and systemic therapy, induced neuronal loss, and this effect may be more severe in older patients with longer treatment history; radiotherapy is known to cause chronic, progressive pathologic changes extending outside the tumor region (33). Direct thalamic radiation effects may play a role in the observed age-related decline. Although radiation dose to the thalamus did not have a significant effect on thalamic AMT uptake, radiation may reduce cortical tryptophan uptake in a dose-dependent manner. On the other hand, thalamic tryptophan uptake was lower in those with longer survival after radiation treatment. Therefore, it is likely that chronic, progressive brain tissue damage after initial radiation contributed to lower values in older posttreatment patients.

Despite the relatively large cohort of glioma patients, our study had some limitations. The analysis was retrospective, and patients in the posttreatment group were scanned at different times after initial treatment. The patients also received variable treatments after the PET scan: for example, some underwent repeated surgery or received antiangiogenic rescue treatment with bevacizumab. However, in our recent study, repeated surgery or bevacizumab treatment after AMT PET were not prognostic (11); also, a recently concluded large bevacizumab trial found no robust drug effects on overall survival (34). Thus, postscan therapy was unlikely to have a major effect on the prognostic results. Another potential prognostic factor of posttreatment survival is performance status. We did not include this factor as a potential predictor, as reliable data were not always available at the time of the PET scan because of the retrospective nature of the study. However, performance status was unlikely to have a robust impact on survival in this group, considering that patients with poor functional status (typically a Karnofsky performance score $<60$ ) were not included in the research PET studies. Likewise, we did not include some molecular markers (such as isocitrate dehydrogenase 1 mutations) in the analysis, because of the lack of complete dataset in this regard. On the basis of the present results, we are now conducting a prospective study in which these potential prognostic factors will be included. In addition, we focused on selected gray matter regions (thalamus and frontal, temporal cortices) contralateral to the tumor. It is possible that some other cortical regions would also show clinical correlates. Some of these regions, including brain structures involved in serotonergic networks, may be important to study to clarify the relevance of the findings for depression or other glioma-related comorbidities in future studies.

\section{CONCLUSION}

In this study, we found posttreatment age-related decline and sex differences in brain tryptophan uptake, present mostly in subjects with a previously treated glioma. Thalamic uptake changes of the nontumoral hemisphere were highly prognostic for posttreatment survival. These findings suggest altered tryptophan metabolism in the nontumoral brain, with potentially important clinical implications for prognosis and comorbidities affecting the serotonergic system.

\section{DISCLOSURE}

The costs of publication of this article were defrayed in part by the payment of page charges. Therefore, and solely to indicate this fact, this article is hereby marked "advertisement" in accordance with 18 USC section 1734. The study was supported by a grant (R01 CA123451) from the National Cancer Institute; a grant from the Fund for Medical Research and Education, Wayne State University School of Medicine; and a Strategic Research Initiative grant from the Karmanos Cancer Institute. No other potential conflict of interest relevant to this article was reported.

\section{ACKNOWLEDGMENTS}

We thank Janet Barger, Kelly Forcucci, and Cathie Germain for assisting with patient recruitment and scheduling. We are grateful to the entire staff at the PET Center, Children's Hospital of Michigan, who provided invaluable technical help in performing the PET scans.

\section{REFERENCES}

1. Diksic M, Nagahiro S, Sourkes TL, Yamamoto YL. A new method to measure brain serotonin synthesis in vivo. I. Theory and basic data for a biological model. J Cereb Blood Flow Metab. 1990;10:1-12.

2. Chugani DC, Muzik O, Chakraborty P, Mangner T, Chugani HT. Human brain serotonin synthesis capacity measured in vivo with alpha-[C-11]methyl-L-tryptophan. Synapse. 1998;28:33-43.

3. Diksic M, Young SN. Study of the brain serotonergic system with labeled

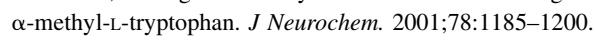

4. Chugani DC, Muzik O. $\alpha[$ C-11]methyl-L-tryptophan PET maps brain serotonin synthesis and kynurenine pathway metabolism. J Cereb Blood Flow Metab. 2000;20:2-9.

5. Zitron IM, Kamson DO, Kiousis S, Juhasz C, Mittal S. In vivo metabolism of tryptophan in meningiomas is mediated by indoleamine 2,3-dioxygenase 1. Cancer Biol Ther. 2013;14:333-339.

6. Batista CE, Juhasz C, Muzik O, et al. Imaging correlates of differential expression of indoleamine 2,3-dioxygenase in human brain tumors. Mol Imaging Biol. 2009;11:460-466.

7. Wainwright DA, Balyasnikova IV, Chang AL, et al. IDO expression in brain tumors increases the recruitment of regulatory $\mathrm{T}$ cells and negatively impacts survival. Clin Cancer Res. 2012;18:6110-6121.

8. Mitsuka K, Kawataki T, Satoh E, Asahara T, Horikoshi T, Kinouchi H. Expression of indoleamine 2,3-dioxygenase and correlation with pathological malignancy in gliomas. Neurosurgery. 2013;72:1031-1038.

9. Juhász C, Chugani DC, Muzik O, et al. In vivo uptake and metabolism of $\alpha-\left[{ }^{11} \mathrm{C}\right]$ methyl-L-tryptophan in human brain tumors. J Cereb Blood Flow Metab. 2006; 26:345-357.

10. Kamson DO, Juhasz C, Buth A, et al. Tryptophan PET in pretreatment delineation of newly-diagnosed gliomas: MRI and histopathologic correlates. J Neurooncol. 2013;112:121-132.

11. Kamson DO, Mittal S, Robinette NL, et al. Increased tryptophan uptake on PET has strong independent prognostic value in patients with a previously treated high-grade glioma. Neuro Oncol. April 1, 2014 [Epub ahead of print].

12. Dhermain FG, Hau P, Lanfermann H, Jacobs AH, van den Bent MJ. Advanced MRI and PET imaging for assessment of treatment response in patients with gliomas. Lancet Neurol. 2010;9:906-920.

13. Gulyás B, Halldin C. New PET radiopharmaceuticals beyond FDG for brain tumor imaging. Q J Nucl Med Mol Imaging. 2012;56:173-190.

14. Rosa-Neto P, Benkelfat C, Sakai Y, Leyton M, Morais JA, Diksic M. Brain regional $\alpha-\left[{ }^{11} \mathrm{C}\right]$ methyl-L-tryptophan trapping, used as an index of 5-HT synthesis, 
in healthy adults: absence of an age effect. Eur J Nucl Med Mol Imaging. 2007; 34:1254-1264.

15. Nishizawa S, Benkelfat C, Young SN, et al. Differences between males and females in rates of serotonin synthesis in human brain. Proc Natl Acad Sci USA. 1997;94:5308-5313.

16. Okazawa H, Leyton M, Benkelfat C, Mzengeza S, Diksic M. Statistical mapping analysis of serotonin synthesis images generated in healthy volunteers using positron-emission tomography and $\alpha-\left[{ }^{11} \mathrm{C}\right]$ methyl-L-tryptophan. J Psychiatry Neurosci. 2000;25:359-370.

17. Chugani DC, Muzik O, Behen M, et al. Developmental changes in brain serotonin synthesis capacity in autistic and nonautistic children. Ann Neurol. 1999; 45:287-295.

18. Chugani DC, Niimura K, Chaturvedi S, et al. Increased brain serotonin synthesis in migraine. Neurology. 1999;53:1473-1479.

19. Frey BN, Skelin I, Sakai Y, Nishikawa M, Diksic M. Gender differences in $\alpha-\left[{ }^{11} \mathrm{C}\right] \mathrm{MTrp}$ brain trapping, an index of serotonin synthesis, in medication-free individuals with major depressive disorder: a positron emission tomography study. Psychiatry Res. 2010;183:157-166.

20. Juhász C, Chugani DC, Barger GR, et al. Quantitative PET imaging of tryptophan accumulation in gliomas and remote cortex: correlation with tumor proliferative activity. Clin Nucl Med. 2012;37:838-842.

21. Chakraborty PK, Mangner TJ, Chugani DC, Muzik O, Chugani HT. A high-yield and simplified procedure for the synthesis of $\alpha-\left[{ }^{11} \mathrm{C}\right]$ methyl-L-tryptophan. Nucl Med Biol. 1996;23:1005-1008.

22. Alkonyi B, Barger GR, Mittal S, et al. Accurate differentiation of recurrent gliomas from radiation injury by kinetic analysis of $\alpha-{ }^{11} \mathrm{C}$-methyl-L-tryptophan PET. J Nucl Med. 2012;53:1058-1064.

23. Kamson DO, Mittal S, Buth A, et al. Differentiation of glioblastomas from metastatic brain tumors by tryptophan uptake and kinetic analysis: a positron emission tomographic study with magnetic resonance imaging comparison. Mol Imaging. 2013;12:327-337.
24. Patlak CS, Blasberg RG, Fenstermacher JD. Graphical evaluation of blood-to-brain transfer constants from multiple-time uptake data. J Cereb Blood Flow Metab. 1983;3:1-7.

25. Fedorov A, Beichel R, Kalpathy-Cramer J, et al. 3D Slicer as an image computing platform for the Quantitative Imaging Network. Magn Reson Imaging. 2012; 30:1323-1341.

26. Mattes D, Haynor DR, Vesselle H, Lewellen TK, Eubank W. PET-CT image registration in the chest using free-form deformations. IEEE Trans Med Imaging. 2003;22:120-128.

27. Tamura M, Ohye C, Nakazato Y. Pathological anatomy of autopsy brain with malignant glioma. Neurol Med Chir (Tokyo). 1993;33:77-80.

28. Iwami K, Natsume A, Wakabayashi T. Cytokine networks in glioma. Neurosurg Rev. 2011;34:253-263.

29. Sakai Y, Nishikawa M, Leyton M, Benkelfat C, Young SN, Diksic M. Cortical trapping of $\alpha-\left[{ }^{11} \mathrm{C}\right]$ methyl-1-tryptophan, an index of serotonin synthesis, is lower in females than males. Neuroimage. 2006;33:815-824.

30. Pringle AM, Taylor R, Whittle IR. Anxiety and depression in patients with an intracranial neoplasm before and after tumour surgery. Br J Neurosurg. 1999;13: 46-51.

31. Rooney AG, Brown PD, Reijneveld JC, Grant R. Depression in glioma: a primer for clinicians and researchers. J Neurol Neurosurg Psychiatry. 2014;85:230-235.

32. Arnold SD, Forman LM, Brigidi BD, et al. Evaluation and characterization of generalized anxiety and depression in patients with primary brain tumors. Neuro Oncol. 2008;10:171-181.

33. Siu A, Wind JJ, Iorgulescu JB, Chan TA, Yamada Y, Sherman JH. Radiation necrosis following treatment of high grade glioma:a review of the literature and current understanding. Acta Neurochir (Wien). 2012;154:191-201.

34. Chinot OL, Wick W, Mason W, et al. Bevacizumab plus radiotherapy-temozolomide for newly diagnosed glioblastoma. N Engl J Med. 2014;370:709-722. 